\title{
Rapid, amplification-based fingerprinting of Mycobacterium tuberculosis
}

\author{
Bonnie B. Plikaytis, ${ }^{1 *}$ Jack T. Crawford, ${ }^{1}$ Charles L. Woodley, ${ }^{1}$ W. Ray Butler, ${ }^{1}$ \\ Kathleen D. Eisenach, ${ }^{2}$ M. Donald CaVE ${ }^{3}$ and Thomas M. Shinnick ${ }^{1}$ \\ ${ }^{1}$ Division of Bacterial and Mycotic Diseases, National Center for Infectious Diseases, Centers for Disease Control and \\ Prevention, Atlanta, GA 30333, USA \\ ${ }^{2,3}$ Departments of Pathology ${ }^{2}$ and Anatomy ${ }^{3}$, University of Arkansas for Medical Sciences, Little Rock, AR 72205, \\ $U S A$
}

(Received 22 October 1992; revised 25 January 1993; accepted 24 February 1993)

\begin{abstract}
Insertion element IS6110 occurs in multiple copies throughout the Mycobacterium tuberculosis genome, and the variability of its insertion sites is the basis for the IS6110 restriction fragment length polymorphism (RFLP) method for typing. We describe a novel gene amplification method to assess the variability of the location of IS6110. A unilateral-nested polymerase chain reaction and hybridization procedure was used to measure the variability in the distances between IS6110 elements and copies of a major polymorphic tandem repeat sequence of $M$. tuberculosis. The pattern of amplicons produced could be used to cluster epidemiologically related strains of $M$. tuberculosis into groups which correlated with the groups formed using IS6110-RFLP typing. Reliable patterns can be generated directly from sputum specimens as well as from $M$. tuberculosis cultures. We designated the novel method as IS6110-ampliprinting.
\end{abstract}

\section{Introduction}

Tuberculosis remains a major source of morbidity and mortality throughout the world and is increasing in the United States today. The resurgence of tuberculosis in the United States is largely related to the human immunodeficiency virus (HIV) epidemic (Barnes et al., 1991). Because of the reduction in cell-mediated immunity in HIV-infected persons, active disease may develop quickly after exposure to Mycobacterium tuberculosis (Barnes et al., 1991). As the number of hospitalized patients infected with HIV and tuberculosis increases, the risk of nosocomial infection with $M$. tuberculosis increases not only among patients, but also among health care providers (Pearson et al., 1992).

An important factor in the control of tuberculosis is the ability to identify outbreaks and track the transmission of a particular strain of $M$. tuberculosis. The standard procedure for distinguishing strains of $M$. tuberculosis isolates has been phage typing; however, a more sensitive molecular approach has been described recently (Hermans et al., 1990; Cave et al., 1991). An insertion element which has considerable homology to

*Author for correspondence. Tel. (404) 639 3601; fax (404) 639 3037.

Abbreviations: MPTR, major polymorphic tandem repeat; RFLP, restriction fragment length polymorphism. the IS3 family of enterobacteria has been described and designated as IS6110 (Thierry et al., 1990); it is virtually identical to the more recently described IS986 (McAdam et al., 1990). This approach takes advantage of the facts that $M$. tuberculosis strains carry multiple copies of IS6110, and that the precise locations of the IS6110 elements in the $M$. tuberculosis genome vary significantly from strain to strain, providing a unique DNA fingerprint for each $M$. tuberculosis strain. The IS6110restriction fragment length polymorphism (RFLP) technique has been shown to be a reliable and reproducible method for differentiating $M$. tuberculosis strains (Cave et al., 1991; Otal et al., 1991), and a recent investigation utilized this technique to study the epidemiology of multidrug-resistant tuberculosis among hospitalized HIV-infected patients (Edlin et al., 1992).

The IS6110-RFLP procedure requires growth of the organism followed by purification of genomic DNA from the bacteria. The purified DNA is digested with a restriction enzyme which cleaves within the IS6110 sequence. The digested genomic DNA is then electrophoresed on agarose gels, transferred to a membrane, and hybridized with a portion of the IS6110 sequence. This is a time-consuming method that is useful for retrospective epidemiology but has limitations concerning disease management or a rapid response to outbreak situations.

We explored the possibility of using a polymerase 
chain reaction (PCR) (Mullis \& Faloona, 1987) and hybridization procedure to measure the variability in the distances between IS6110 elements and a major polymorphic tandem repeat (MPTR) sequence of $M$. $t u$ berculosis (Hermans et al., 1992; Shinnick, 1987). The MPTR sequence is composed of tandem repeats of a $10 \mathrm{bp}$ consensus sequence separated by $5 \mathrm{bp}$ highly heterogeneous spacers. The consensus sequence of the $10 \mathrm{bp}$ tandem repeat is $\left(5^{\prime}\right)$ GCCGGTGTTG, which has homology to the repetitive extragenic palindromic (REP) sequences of Escherichia coli (Hermans et al., 1992). REP sequences have been used to produce PCR-based fingerprints from various bacterial genomes (Versalovic et al., 1991). The MPTR sequences are found in the $M$. tuberculosis genome in as many as 80 different regions (Hermans et al., 1992). Importantly, their distribution in the $M$. tuberculosis genome appears to be stable, in contrast to the variability in IS6110 location (Hermans et al., 1992). Thus, by using IS6110 and MPTR primers in a unilateral-nested amplification procedure followed by hybridization with an IS6110-specific oligonucleotide, we were able to generate a pattern of amplification products that clustered strains of $M$. tuberculosis into groups matching those formed using the IS6110-RFLP technique. As a test of the procedure, we analysed strains from several recent multidrug-resistant tuberculosis outbreaks. We also tested the method on a limited number of sputum samples to determine the feasibility of fingerprinting $M$. tuberculosis directly from sputum specimens (i.e. without culturing). We refer to this method as ampliprinting.

\section{Methods}

Bacterial strains and preparation of DNA. The strains used in this study are listed in Table 1. DNA was prepared from $M$. tuberculosis isolates using the CTAB (cetyltrimethylammonium bromide) method as previously described (Wilson, 1990). Crude lysates of the non- $M$. tuberculosis strains listed in Table 1 were prepared by glass bead lysis as previously described (Plikaytis et al., 1992).

Preparation of lysates from sputum specimens. Smear-positive sputum samples were obtained from the Arkansas State Health Department laboratory. The specimens were liquefied and decontaminated using the standard $n$-acetylcysteine/ $\mathrm{NaOH}$ protocol (Kent \& Kubica, 1985), and the sediments remaining after microscopy and inoculation on solid media were stored at $-20^{\circ} \mathrm{C}$ until they were processed for PCR. The sediment was centrifuged at $16000 \mathrm{~g}$ for $5 \mathrm{~min}$ and the supernatant was discarded. The pellet was resuspended in $200 \mu \mathrm{l}$ of $10 \mathrm{~mm}$-Tris $/ \mathrm{HCl}$ (pH 8.0)-1 mM-EDTA-10 mM-NaCl and mixed with $200 \mu 1$ siliconized $0.1 \mathrm{~mm}$-diameter glass beads and $100 \mu \mathrm{l}$ chloroform. The mixture was homogenized for $2 \mathrm{~min}$ at room temperature in a Mickle apparatus (Brinkman Instruments, Westbury, NY, USA) to disrupt the cells. The homogenized suspension was centrifuged at $16000 \mathrm{~g}$ for $5 \mathrm{~min}$, and the aqueous supernatant was transferred to a fresh tube and boiled for $10 \mathrm{~min}$.

Oligonucleotide primers. Primers corresponding to portions of the $M$. tuberculosis IS 6110 and MPTR sequences were synthesized on a DNA synthesizer (model 381A; Applied Biosystems) at the Biotechnology Core Facility, Centers for Disease Control. The sequences and locations of these primers are listed in Table 2, and the relative positions within the IS6110 sequences are depicted in Fig. 1. The MPTR-primer sequences were determined by combining the consensus sequence for the $10 \mathrm{bp}$ repeat with five flanking bases, which were selected by comparing the reported sequences (Hermans et al., 1992; Shinnick, 1987) and identifying 5 bp spacer sequences which appeared only once in each reported sequence. For example, the primer MPTR-6 is composed of the consensus sequence flanked by two bases on the $5^{\prime}$-end and a unique set of three bases on the $3^{\prime}$-end (Table 2).

Gene amplification. The amplification reaction contained $10 \mu \mathrm{l}$ of template DNA and $90 \mu 1$ of a reaction mix [200 $\mu$ M (each) deoxynucleotide triphosphates, $1.0 \mu \mathrm{M}$ (each) primers, $2.5 \mathrm{U} \mathrm{Taq}$ polymerase, $10 \mathrm{~mm}$-Tris $/ \mathrm{HCl}$ (pH 8.3), $50 \mathrm{~mm}-\mathrm{KCl}, 1.5 \mathrm{~mm}-\mathrm{MgCl}_{2}, 0.01 \%$ gelatin] as recommended by the Taq polymerase manufacturer (Perkin-Elmer Cetus). The first round of amplification with an IS6110 primer and an MPTR primer consisted of 25-30 cycles in a programmable thermal cycler (Perkin-Elmer Cetus) with a three-step cycle of denaturation for $1.5 \mathrm{~min}$ at $94{ }^{\circ} \mathrm{C}$, annealing for $1.75 \mathrm{~min}$ at $45^{\circ} \mathrm{C}$, and extension for $2.5 \mathrm{~min}$ at $72{ }^{\circ} \mathrm{C}$. The second round of amplification was carried out for $15-25$ cycles after transferring $10 \%$ of the first-round amplification

Table 1. Strains used in this study

\begin{tabular}{|c|c|c|}
\hline Species & Strain & Fingerprint type* \\
\hline M. tuberculosis & $91-8358,91-8359,91-8360$ & $011-8114$ \\
\hline M. tuberculosis & $\begin{array}{l}91-8361,91-2627,91-2742, \\
91-2743,91-2744,91-2745, \\
91-2746,92-8081,92-8082, \\
92-8083,92-8088,92-8089, \\
92-8090,92-8091,92-8092, \\
92-8093,92-8095\end{array}$ & 021-2072 \\
\hline M. tuberculosis & $91-8271,92-8272,91-8273$ & $023-8271$ \\
\hline M. tuberculosis & $91-8275,91-8276$ & $024-8275$ \\
\hline M. tuberculosis & $92-8078,82-8079$ & $036-8078$ \\
\hline M. tuberculosis & $92-8084,92-8085,92-8086$ & 036-8084 \\
\hline M. tuberculosis & $91-3048$ & 028-3048 \\
\hline M. tuberculosis & $91-3054$ & $028-3054$ \\
\hline M. tuberculosis & $91-8309$ & 031-8309 \\
\hline M. tuberculosis & $91-8310$ & 031-8310 \\
\hline M. tuberculosis & $91-8311$ & $031-8311$ \\
\hline M. tuberculosis & $91-8312$ & $031-8312$ \\
\hline M. tuberculosis & $91-8313$ & $031-8313$ \\
\hline M. tuberculosis & $91-8270$ & $023-8270$ \\
\hline M. tuberculosis & $91-8277$ & $024-8277$ \\
\hline M. tuberculosis & $\mathrm{H} 37 \mathrm{Rv}$ & $\mathrm{H} 37 \mathrm{Rv}$ \\
\hline M. bovis & TMC 401, TMC 410 & \\
\hline M. bovis $\mathrm{BCG}$ & TMC 1024 & \\
\hline M. africanum & TMC 5122 & \\
\hline M. avium & TMC 1461 & \\
\hline M. chelonae & TMC 1524 & \\
\hline M. fortuitum & TMC 1530 & \\
\hline M. gastri & ATCC 25157 & \\
\hline M. gordonae & TMC 1325 & \\
\hline M. intracellulare & TMC 1469 & \\
\hline M. kansasii & ATCC 12478 & \\
\hline M. scrofulaceum & TMC 1312 & \\
\hline M. smegmatis & TMC 1533 & \\
\hline M. szulgai & $91-698$ & \\
\hline
\end{tabular}

* The IS 6110 -RFLP fingerprint type consists of the outbreak or study number followed by the isolate number. All subsequent isolates having that identical fingerprint pattern are given the same type designation as the initial isolate having that pattern. 
Table 2. Sequences of oligonucleotides used as primers or hybridization probes

\begin{tabular}{cclc}
\hline \hline Primer & Target & \multicolumn{1}{c}{ Sequence $\left(5^{\prime}-3^{\prime}\right)$} & Location* $^{*}$ \\
\hline IS49 & IS6110 & CATGTCAGGTGGTTCATCG & $54-72$ \\
IS54 & IS6110 & TCGACTGGTTCAACCATCGCCG & $1210-1231$ \\
IS55 & IS6110 & TCTGATCTGAGACCTCAGC & $1330-1312 \mathrm{c}$ \\
IS56 & IS6110 & GCGACCTCACTGATCGCTGC & $170-151 \mathrm{c}$ \\
IS61 & IS6110 & GACCGCGGATCTCTGCGACC & $133-114 \mathrm{c}$ \\
IS62 & IS6110 & ACCAGTACTGCGGCGACGTC & $1237-1256$ \\
MPTR-1 & MPTR & GCCGGTGTTGGTGTC & $154-168 \dagger$ \\
MPTR-6 & MPTR & GGCAACACCGGCCTC & $255-241 \mathrm{c} \dagger$ \\
\hline \hline
\end{tabular}

* The residues of IS6110 are numbered according to the system of Thierry et al. (1990), and those of MPTR according to Hermans et al. (1992). A ' $c$ ' indicates that the listed sequence is the complement of the published sequence.

$\dagger$ The primer sequence for MPTR-1 and MPTR- 6 includes the consensus sequence (GCCGGTGTTG) instead of the actual sequences for these residues, which are CCCGGTGTTGGTGTC (154-165) and GGCAGCACTGGGCTC (255-241c).

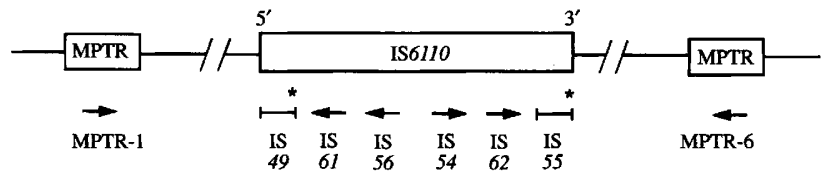

Fig. 1. Schematic representation of IS 6110 and MPTR sequences and the location of oligonucleotides used in PCR and Southern blotting. * denotes the ECL fluorescein-labelled oligonucleotide used as a probe in the Southern blot.

mixture into a fresh tube containing reaction mixture with a nested IS6110 primer and the same MPTR primer as used in the first round. The second round of amplification used a three-step cycle similar to that used in the first round, except that the annealing temperature was $60^{\circ} \mathrm{C}$.

Hybridization. Fifteen microlitres of the second-round amplification mixture were electrophoresed on a $1.5 \%$ agarose gel, and the reaction products were visualized by ethidium bromide fluorescence. The DNA was then denatured, neutralized, and transferred by capillary blotting to Hybond- $\mathrm{N}^{+}$membrane (Amersham) as recommended by the manufacturer of the membrane. DNA was bound to the membrane using a Stratalinker UV crosslinker (Stratagene). An IS6110 (IS49 or IS55) or MPTR (MPTR-6) specific oligonucleotide was labelled using the ECL 3'-oligolabelling and detection system (Amersham). The membrane was hybridized according to the manufacturer's recommendations at $42{ }^{\circ} \mathrm{C}$ for $3 \mathrm{~h}$ and washed stringently at $42^{\circ} \mathrm{C}$ in $3 \mathrm{~mm}$ sodium citrate, $30 \mathrm{~mm}$-sodium chloride and $0.1 \%$ sodium dodecyl sulphate. After development with the ECL detection solutions, the signals were detected using X-OMAT AR autoradiography film (Eastman Kodak).

\section{Results and Discussion}

\section{Evaluation of primers for $M$. tuberculosis amplified} fingerprinting (ampliprinting)

Two sets of IS6110-specific primers, one at the left (IS56, IS61, IS49) and one at the right (IS54, IS62, IS55) end were evaluated. Each set contained two nested primers for amplification and a third for use as a hybridization probe (Fig. 1). In the first round of amplification a $45^{\circ} \mathrm{C}$ annealing temperature was used to allow for potential mismatches in the slightly heterogeneous MPTR consensus sequence. The second round, which is a unilateralnested amplification since the same primer is used for the MPTR sequence, employs a more stringent annealing temperature of $60^{\circ} \mathrm{C}$. The use of two IS6110-specific primers and the increased annealing temperature improves the efficiency of amplification of IS6110-containing sequences. Initially six MPTR primers were tested with each of the two sets of IS6110 primers. MPTR-1 was found to produce the most informative patterns when paired with the IS6110 left set of primers, and MPTR-6 was found to be best for use with the IS6110 right set (data not shown). These primers and the IS6110 primers were then used individually and in combination with each other to characterize further the amplification patterns produced from $M$. tuberculosis. That is, strain 91-8361 DNA was amplified using IS56 alone, MPTR-1 alone, IS56 + MPTR-1 (first round), and IS56 + MPTR-1 followed by IS61 + MPTR-1 (second round), or using IS54 alone, MPTR-6 alone, IS54 + MPTR-6, and IS54+MPTR-6 followed by IS62 + MPTR-6. DNA fragments ranging in size from approximately 250 to $2200 \mathrm{bp}$ were amplified by each primer individually or by each combination of primers tested (Fig. 2a,c). To enhance the signal from the IS6110-containing amplicons, the samples were hybridised with an IS6110-specific oligonucleotide probe (Fig. $2 b, d)$. The hybridization step eliminated the signals from the products amplified due to mispriming or due solely to the MPTR primer (lane 2, Fig. $2 b, d$ ) and produced a discrete pattern of 9 to 11 IS6110-containing amplicons (lane 4, Fig. 2b,d).

\section{Comparison of $5^{\prime}$-end and $3^{\prime}$-end primer sets}

To determine which set of primers was the most useful or if data from both sets would be necessary to distinguish different strains of $M$. tuberculosis, 16 strains were 


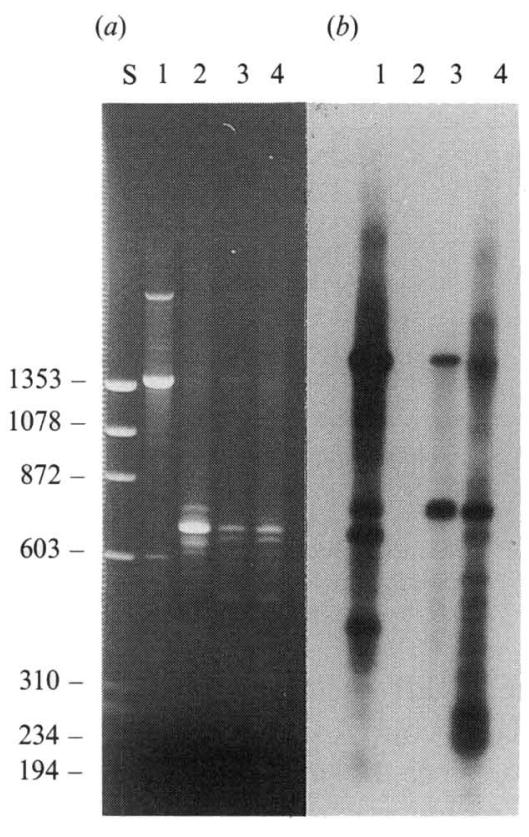

(a)

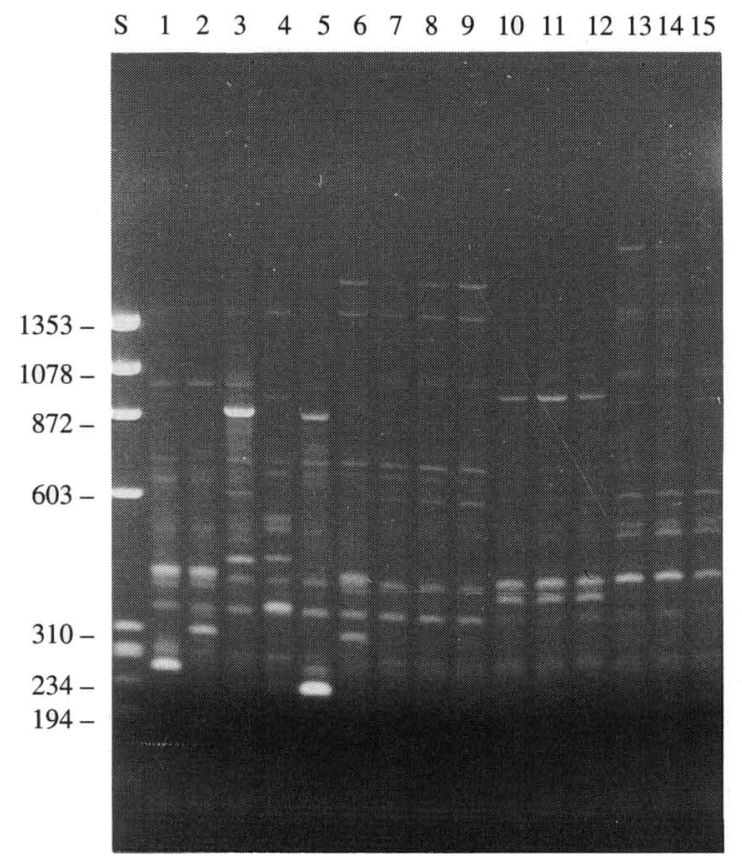

(c)

$$
\begin{array}{llllllll}
5 & 6 & 7 & 8 & 5 & 6 & 7 & 8
\end{array}
$$

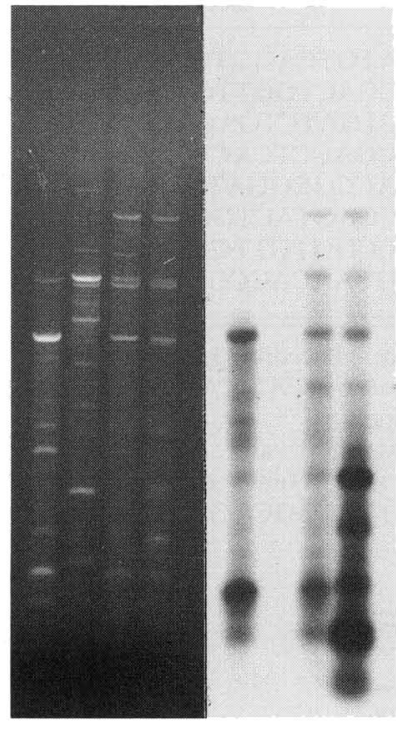

Fig. 2. Amplification with IS6110 and MPTR primers individually and in combinations. The oligonucleotide primer(s) used to amplify DNA from a multidrug-resistant strain of $M$. tuberculosis, 91-8361, were IS56 only (lane 1), MPTR-1 only (lane 2), IS56 + MPTR-1 (lane 3), IS56 + MPTR-1 followed by IS61+MPTR-1 (lane 4), IS54 only (lane 5), MPTR-6 only (lane 6), IS54 + MPTR-6 (lane 7), IS54 + MPTR-6 followed by IS $62+$ MPTR-6 (lane 8 ). Panel $(b)$ is the Southern blot analysis of the agarose gel shown in panel $(a)$ hybridized with the IS6110 5'-end oligonucleotide IS49. Panel $(d)$ is the Southern blot analysis of the agarose gel shown in panel (c) hybridized with the IS $61103^{\prime}$-end oligonucleotide IS55. Lane $\mathrm{S}$ contains molecular size markers ( $\phi$ X174RF DNA/HaeIII fragments), with the sizes in bp indicated on the left.

(b)

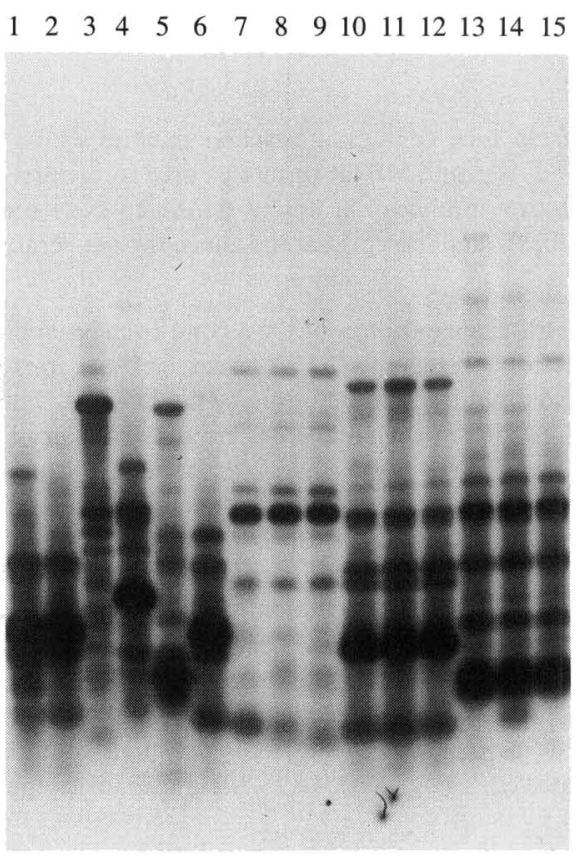

Fig. 3. Evaluation of the $3^{\prime}$-end primer set. (a) Agarose gel electrophoresis and (b) Southern blot analysis showing the ampliprints generated using primers IS54+MPTR-6 in the first round of amplification followed by IS62+MPTR-6 in the second round of amplification. DNA templates were $M$. tuberculosis isolates of 91-8309 (lane 1), 91-8313 (lane 2), 91-8310 (lane 3), 91-8312 (lane 4), 913054 (lane 5), 91-8270 (lane 6), 91-8271 (lane 7), 91-8272 (lane 8), 91-8273 (lane 9), 91-8358 (lane 10), 91-8359 (lane 11), 91-8360 (lane 12), 92-8082 (lane 13), 92-8093 (lane 14), 92-8095 (lane 15). Lane $S$ contains the molecular size markers ( $\phi X 174 \mathrm{RF}$ DNA/HaeIII fragments), with the sizes in bp indicated on the left.

amplified using primer sets IS56 + MPTR-1 (5'-end) and IS54 + MPTR-6 (3'-end) in the first round followed by a second round of amplification with IS61 + MPTR-1 (5'end) and IS62 + MPTR-6 (3'-end) and then were hybrid- ized with the corresponding probe. The 16 strains analysed represent six distinct IS6110-RFLP types (J. T. Crawford, unpublished data). Each set of primers generated a distinctive pattern of IS6110-containing 
amplicons for each of the six IS6110-RFLP types (data not shown). The 3'-end primer set was chosen for further study because it appeared to give a slightly more reproducible pattern.

\section{Evaluation of the 3'-end primer set}

Purified DNA from $M$. tuberculosis H37Rv was titrated to determine the detection limit of the method using 30 cycles of amplification in the first round of amplification and 15 cycles in the second round of amplification. The pattern of IS6110-containing amplicons was maintained to $100 \mathrm{pg}$ of DNA, which is equivalent to approximately $2 \times 10^{4}$ organisms (data not shown). At smaller amounts of DNA, the major PCR products were still amplified; however, several of the minor amplification products were not. This reduces the amount of information in the patterns, which, in turn, may lessen the ability of the method to differentiate strains.

The specificity of the method was evaluated by amplifying crude lysates of $M$. africanum, $M$. avium, $M$. bovis, $M$. bovis BCG, M. chelonae, $M$. fortuitum, $M$. gastri, $M$. gordonae, $M$. intracellulare, $M$. kansasii, $M$. scrofulaceum, $M$. smegmatis and $M$. szulgai. Various numbers of ethidium-bromide-stained fragments were amplified from each species. However, only the $M$. africanum sample contained amplicons that hybridized with the IS6110-specific IS55 oligonucleotide (data not shown). The positive hybridization results with $M$. africanum are expected since this strain contains 6 to 9 copies of the IS6110 sequence (Plikaytis et al., 1991). The negative hybridization results with $M$. bovis and $M$. bovis BCG are not surprising, however, since there are only 1 to 3 copies of the IS 6110 sequence in the $M$. bovis genome.

Many fragments in the amplified samples from each of the Mycobacterium species tested hybridized to MPTR6 (data not shown). This suggests that sequences homologous to the MPTR consensus sequence are present in the genome of each of these species. However, these amplification results do not distinguish clusters of tandem repeats from fortuitous positioning of individual sequences homologous to the MPTR primer. The latter may be important because a Southern hybridization analysis of genomic DNA suggested that clusters of homologous tandem repeats were found in only $M$. tuberculosis complex species, $M$. gastri, M. gordonae, $M$. kansasii and M. szulgai (Hermans et al., 1992).

\section{Clinical isolates and sputum specimens}

Type strain H37Rv and 39 M. tuberculosis clinical isolates from seven tuberculosis outbreak investigations representing 16 IS6110-RFLP types were analysed with

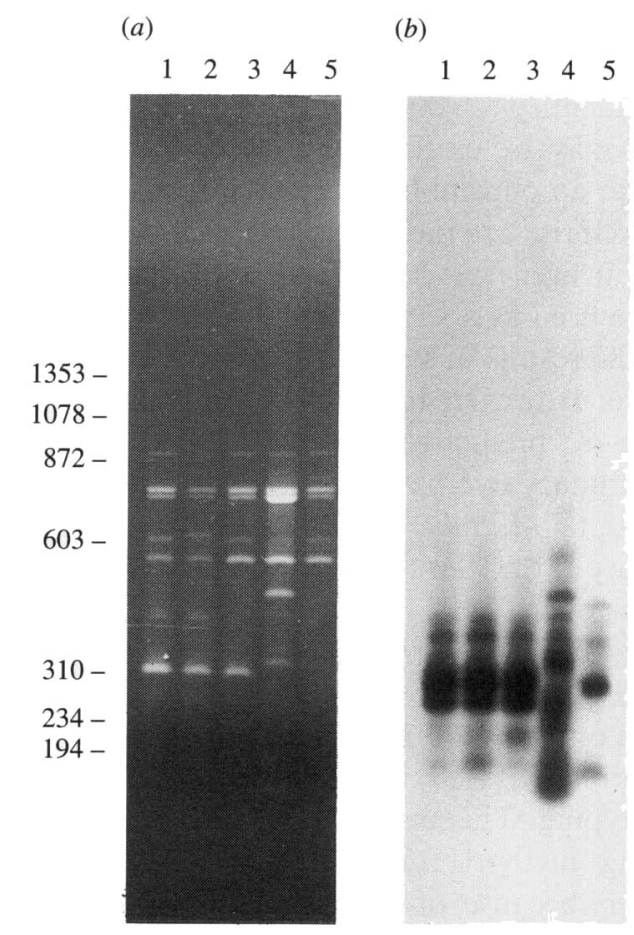

Fig. 4. Ampliprints obtained directly from sputum samples. (a) Agarose gel electrophoresis and $(b)$ a Southern blot showing the ampliprints generated using primers IS54+MPTR-6 in the first round of amplification followed by IS62+MPTR-6 in the second round of amplification. DNA templates were crude lysates of organisms obtained directly from sputum samples from tuberculosis patient $\mathrm{D}$ : specimens 11923, 11925 and 13066 (lanes 1, 2 and 3); patient E: specimen 11770 (lane 4); and patient F: specimen 12907 (lane 5). The sizes in bp of the molecular size standards ( $\phi$ X174RF DNA/HaeIII fragments) are indicated on the left.

the $3^{\prime}$-end primer set. Fig. 3(a) shows the agarose gel containing the products from the second round of amplification of 15 of these $M$. tuberculosis strains representing nine IS6110-RFLP types, and Fig. 3(b) shows the hybridization of these products with the 3 -end probe, IS55. Each of the 16 IS6110-RFLP types produced a unique set of IS6110-containing amplicons. Furthermore, strains that had identical IS6110-RFLP patterns also had identical IS6110-ampliprint patterns (e.g. lanes 7 to 9 , Fig. 3).

Six smear-positive ( 3 to $4+$ ) sputum specimens from three tuberculosis patients were processed as described in Methods, and the crude lysates were amplified for 30 cycles in the first round and 25 cycles in the second round using the $3^{\prime}$-end primers. Fig. 4 shows the agarose gel and the results of the hybridization with the IS55 probe of five of these samples from the three patients. As would be expected for epidemiologically unrelated cases, the ampliprints generated from each patient's specimens were clearly different, while identical hybridization patterns were observed for each of the three serial specimens from one patient (e.g. lanes 1 to 3, Fig. $4 b$ ). 
The ability to type strains directly from sputum specimens suggests that the method could be used to rapidly identify infections with a given strain. If so, this method may be useful in outbreak situations where a particular ampliprint has been identified and strains with this ampliprint are multidrug-resistant. In such cases the ampliprint generated by this amplification procedure might be used as a surrogate marker for drug resistance before the results of standard susceptibility tests become available. However, much work remains to be done to evaluate the predictive value of such an approach.

In summary, we have described a novel approach to differentiate $M$. tuberculosis strains by combining the variability of the IS6110 insertion sites with the conserved locations of the MPTR sequences in the M. tuberculosis genome. Primers corresponding to portions of these sequences were used to amplify a set of DNA fragments that can differentiate $M$. tuberculosis strains into groups that correlated well with those obtained by IS6110RFLP typing. This method should be useful as a rapid screening method for fingerprinting $M$. tuberculosis. However, because of the limited number and sizes of amplicons produced, and the resulting limited information, ampliprinting may provide somewhat less information about strain relatedness than the IS6110RFLP fingerprinting method.

\section{References}

Barnes, P. F., Bloch, A. B., Davidson, P. T. \& Snider, D. E., JR (1991). Tuberculosis in patients with human immunodeficiency virus infection. New England Journal of Medicine 324, 1644-1650.

Cave, M. D., Eisenach, K. D., McDermott, P. F., Bates, J. H. \& CRAWFord, J. T. (1991). IS6110: conservation of sequence in the Mycobacterium tuberculosis complex and its utilization in DNA fingerprinting. Molecular and Cellular Probes 5, 73-80.

Edlin, B. R., Tokars, J. I., Grieco, M. H., Crawford, J. T., Williams, J., Sordillo, E. M., ONG, K. R., Kilburn, J. O., DoOley, S. W., Castro, K. G., JaR vis, W. R. \& Holmberg, S. D. (1992). An outbreak of multidrug-resistant tuberculosis among hospitalized patients with the acquired immunodeficiency syndrome. New England Journal of Medicine 326, 1514-1521.
Hermans, P. W. M., van Soolingen, D., Dale, J. W., Schuitema, A. R. J., McAdam, R. A., Catty, D. \& van Embden, J. D. A. (1990). Insertion element IS986 from Mycobacterium tuberculosis: a useful tool for diagnosis and epidemiology of tuberculosis. Journal of Clinical Microbiology 28, 2051-2058.

Hermans, P. W. M., van Soolingen, D. \& van Embden, J. D. A. (1992). Characterization of a major polymorphic tandem repeat in Mycobacterium tuberculosis and its potential use in the epidemiology of Mycobacterium kansasii and Mycobacterium gordonae. Journal of Bacteriology 174, 4157-4165.

Kent, B. B. \& KubICA, K. G. P. (1985). Public Health Mycobacteriology: a Guide for the Level III Laboratory. Atlanta, Georgia: US Department of Health and Human Services, Centers for Disease Control.

McAdam, R. A., Hermans, P. W. M., van Soolingen, D., Zainuddin, A. F., Catty, D., van Embden, J. D. A. \& Dale, J. W. (1990). Characterization of a Mycobacterium tuberculosis insertion sequence belonging to the IS3 family. Molecular Microbiology 4, 1607-1613.

Mullis, K. B. \& FAlOONA, F. A. (1987). Specific synthesis of DNA in vitro via a polymerase-catalyzed chain reaction. Methods in Enzymology 155, 335-350.

Otal, I., Martin, C., Vincent-Levy-Frebault, V., Thierry, D. \& GICQUEL, B. (1991). Restriction fragment length polymorphism analysis using IS6110 as an epidemiological marker in tuberculosis. Journal of Clinical Microbiology 29, 1252-1254.

Pearson, M. L., Jereb, J. A., Frieden, T. R., Crawford, J. T., Davis, B. J., Dooley, S. W. \& JARVIS, W. R. (1992). Nosocomial transmission of multidrug-resistant Mycobacterium tuberculosis: a risk to patients and healthcare workers. Annals of Internal Medicine 117, 191-196.

Plikaytis, B. B., Eisenach, K. D., Crawford, J. T. \& Shinnick, T. M. (1991). Differentiation of Mycobacterium tuberculosis and Mycobacterium bovis by a polymerase chain reaction assay. Molecular and Cellular Probes 5, 215-219.

Plikaytis, B. B., Plikaytis, B. D., Yakrus, M. A., Butler, W. R., Woodley, C. L., Silcox, V. A. \& SHINNICK, T. M. (1992). Differentiation of slowly growing Mycobacterium species, including $M y c o-$ bacterium tuberculosis, by gene amplification and restriction fragment length polymorphism analysis. Journal of Clinical Microbiology 30, 1815-1822.

SHINNICK, T. M. (1987). The 65-kilodalton antigen of Mycobacterium tuberculosis. Journal of Bacteriology 169, 1080-1088.

Thierry, D., CaVe, M. D., Eisenach, K. D., Crawford, J. T., Bates, J. H., GiCQuel, B. \& GueSDon, J. L. (1990). IS6110 an IS-like element of Mycobacterium tuberculosis complex. Nucleic Acids Research 18, 188.

Versalovic, J., KoeUth, T. \& LuPSKI, J. R. (1991). Distribution of repetitive DNA sequences in eubacteria and application to fingerprinting of bacterial genomes. Nucleic Acids Research 19, 6823-6831.

WiLson, K. (1990). Preparation of genomic DNA from bacteria. In Current Protocols in Molecular Biology, vol. 1, pp. 2.4.1-2.4.2. Edited by F. M. Ausubel, R. Brent, R. E. Kinston, D. D. Moore, I. G. Seidman, J. A. Smith \& K. Struhl. New York: Wiley Interscience. 